This item was submitted to Loughborough's Research Repository by the author.

Items in Figshare are protected by copyright, with all rights reserved, unless otherwise indicated.

\title{
A new block based time-frequency approach for underdetermined blind source separation
}

PLEASE CITE THE PUBLISHED VERSION

PUBLISHER

(c) IEEE

VERSION

VoR (Version of Record)

LICENCE

CC BY-NC-ND 4.0

\section{REPOSITORY RECORD}

Chambers, Jonathon, Yuhui Luo, and Sangarapillai Lambotharan. 2019. "A New Block Based Time-frequency Approach for Underdetermined Blind Source Separation”. figshare. https://hdl.handle.net/2134/5752. 
This item was submitted to Loughborough's Institutional Repository (https://dspace.lboro.ac.uk/) by the author and is made available under the following Creative Commons Licence conditions.

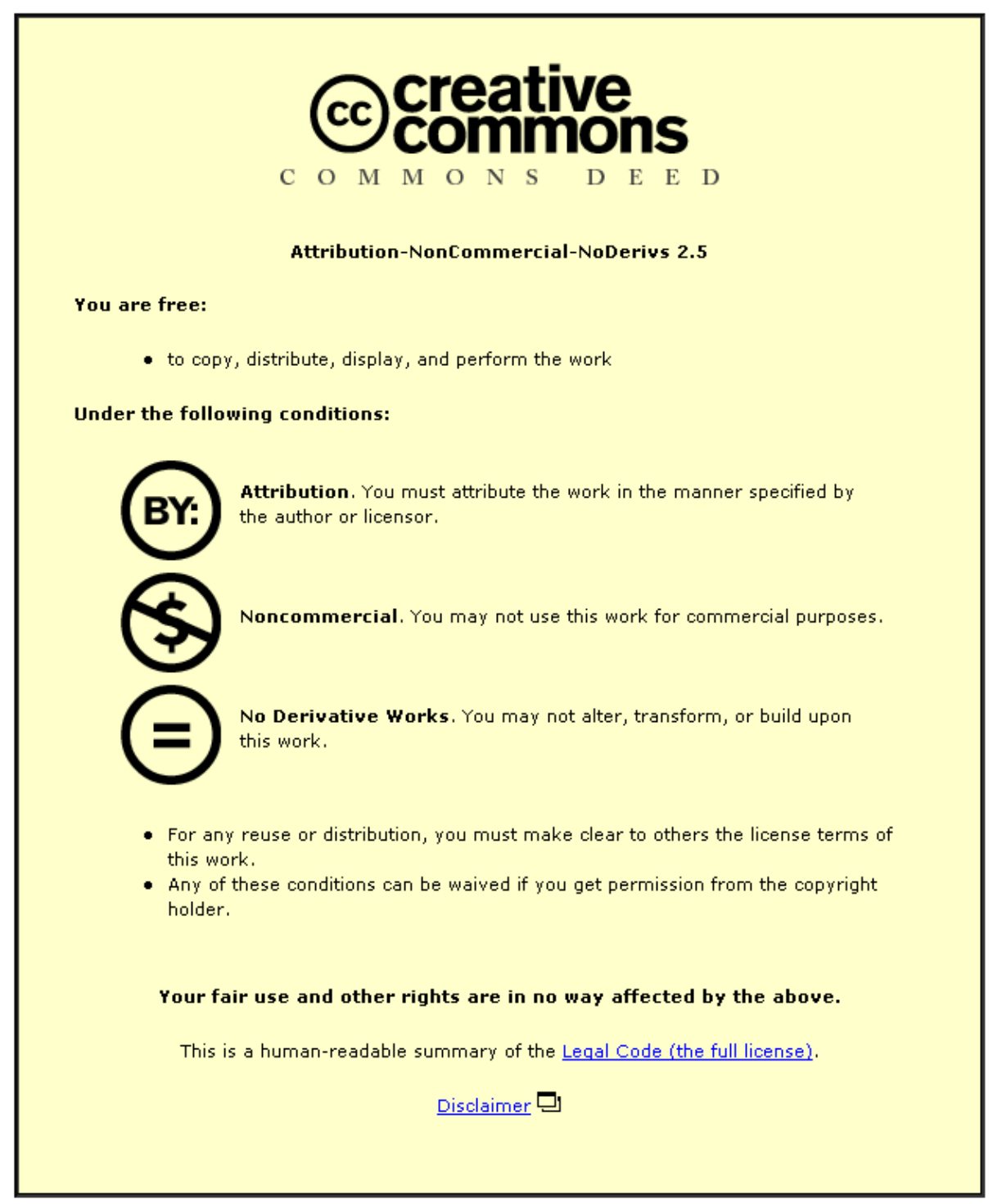

For the full text of this licence, please go to: http://creativecommons.org/licenses/by-nc-nd/2.5/ 


\title{
A NEW BLOCK BASED TIME-FREQUENCY APPROACH FOR UNDERDETERMINED BLIND SOURCE SEPARATION
}

\author{
Y. Luo, S. Lambotharan and J. A. Chambers \\ Centre for Digital Signal Processing Research, \\ Division of Engineering, King's College London, Strand, London, WC2R 2LS. \\ Email: Yuhui.Luo@kcl.ac.uk
}

\begin{abstract}
The problem of underdetermined blind source separation is addressed. The sparse assumption which is commonly required in the current underdetermined blind source separation literature is relaxed. By introducing an advanced clustering technique based upon self-splitting competitive learning, the time-frequency plane is partitioned into appropriate blocks where the number of active sources is no more than the number of sensors, resulting in a novel robust block based algorithm. Simulation studies are presented to support the proposed approach for the separation of GMSK sources.
\end{abstract}

\section{INTRODUCTION}

Given a set of $m$ antennae measurements, the aim of blind source separation (BSS) is to extract the underlying $k$ unknown sources when the transmission channels between the sources and the antennae are also unknown. In data modelling, the antenna measurements are often represented as linear instantaneous mixtures of input sources. Denote $\mathbf{s}(t)=\left[s_{1}(t) s_{2}(t) \ldots s_{k}(t)\right]^{T}$ as the source vector, where $(\cdot)^{T}$ is the transpose operator. The measurement signal $\mathbf{x}(t)=\left[x_{1}(t) x_{2}(t) \ldots x_{m}(t)\right]^{T}$ at discrete time $t$ is written as

$$
\mathbf{x}(t)=A \mathbf{s}(t)+\mathbf{n}(t)
$$

where $A=\left[\mathbf{a}_{1} \ldots \mathbf{a}_{k}\right]$ is an $m \times k$ mixing matrix, $\mathbf{a}_{i}$ is the steering vector of source-i and $\mathbf{n}(t)$ is the zero mean additive noise vector.

Two assumptions are conventionally required in BSS. One is the statistical mutual independence assumption of the sources, which underlies the field of independent component analysis. As independence is lost after linear mixing, an unmixing matrix $B$ of dimension $k \times m$ is introduced. Its output $\mathbf{y}(t)=B \mathbf{x}(t)$ is the estimator of the input sources subject to possible scaling and permutational ambiguities. The second assumption in BSS requires that the mixing matrix $A$ is full column rank. In other words, the number of measurement antennae $m$ should be at least as many as the number of input sources $k$. When this assumption is violated (i.e., $m<k$ ), the problem becomes ill-conditioned and is termed underdetermined BSS (UBSS) in this paper.

So far, most of the UBSS algorithms exploit various kinds of sparsity of the input sources either in the time domain or in the joint time-frequency (t-f) domain. In [3], the FOCUSS algorithm is proposed. It is subsequently suggested for use in UBSS. However a unique solution is guaranteed only if there is a single source present in the system at every time instance. On the basis that the source distribution is assumed sparse, another algorithm which jointly estimates the mixing matrix and the sources is proposed in [1]. Alternatively, by noticing that the received signals will be colinear with the corresponding steering vector if only a single source is present at a given time instant, an approach is proposed in [5]. Recently, the techniques of t- $\mathrm{f}$ analysis have been suggested in the BSS literature, as they are able to reveal the information embedded within the nonstationary signals and are advantageous in the environment of low signal to noise ratio. For the application in UBSS, by assuming that the $t-f$ representation of input sources do not overlap, a method for separating an arbitrary number of sources from two mixtures is proposed in [4]. Also under this t-f orthogonality assumption, the algorithm in [6] separates different sources by clustering the $t-f$ points that are associated with the same steering vectors. However, as the sparse assumption of the input sources may not hold in many practical applications, we are motivated to investigate the situation where the t-f orthogonality assumption is relaxed. By introducing a classification technique called self-splitting competitive learning (SSCL) [7] to divide the t-f plane into appropriate blocks, a new block-based t-f algorithm is proposed for UBSS.

\section{BLOCK BASED TIME-FREQUENCY UNDERDETERMINED BSS ALGORITHM}

To exploit the information present within non-stationary sources in the $\mathrm{t}-\mathrm{f}$ domain, a quadratic $\mathrm{t}-\mathrm{f}$ representation is employed. Denote $\phi(v, l)$ as the signal-independent kernel 
function. The discrete-time form of Cohen's class of t-f representation for antennae signal $x_{i}(t)$, which corresponds to the auto term in the quadratic $\mathrm{t}-\mathrm{f}$ representation, is given by

$$
\begin{aligned}
D_{x_{i} x_{i}}(t, f)= & \sum_{l=-\infty}^{\infty} \sum_{v=-\infty}^{\infty} \phi_{i i}(v, l) \times \\
& x_{i}(t+v-l) x_{i}^{*}(t+v+l) e^{-j 4 \pi f l}
\end{aligned}
$$

The cross t-f representation of two signals $x_{i}(t)$ and $x_{j}(t)$ is written as

$$
\begin{aligned}
D_{x_{i} x_{j}}(t, f)= & \sum_{l=-\infty}^{\infty} \sum_{v=-\infty}^{\infty} \phi_{i j}(v, l) \times \\
& x_{i}(t+v-l) x_{j}^{*}(t+v+l) e^{-j 4 \pi f l}
\end{aligned}
$$

The extension of the $\mathrm{t}-\mathrm{f}$ representation to accommodate vector signals leads to

$$
\begin{aligned}
D_{\mathbf{x x}}(t, f)= & \sum_{l=-\infty}^{\infty} \sum_{v=-\infty}^{\infty} \phi(v, l) \times \\
& \mathbf{x}(t+v-l) \mathbf{x}^{H}(t+v+l) e^{-j 4 \pi f l}
\end{aligned}
$$

where $\phi(v, l)$ is a matrix whose $(i, j)^{t h}$ entry $\phi_{i j}(v, l)$ is the kernel associated with the $i^{\text {th }}$ and the $j^{\text {th }}$ measurement sensor output. Note that $D_{\mathbf{x x}}(t, f)$ is related to that of the input sources by the following key equation

$$
D_{\mathbf{x x}}(t, f)=A D_{\mathbf{s s}}(t, f) A^{H}
$$

In [6], under the t-f orthogonality assumption, $D_{\mathbf{x x}}(t, f)$ is a rank-1 matrix at every t-f point $\left(t_{i}, f_{i}\right)$. Signal separation therefore becomes possible by clustering the t-f points associated with same steering vector, which can readily be estimated with the principle eigenvector of $D_{\mathbf{x x}}(t, f)$.

For better practicality, in this work, we relax the t-f orthogonal assumption such that

Assumption 1. At any time instant, the number of active sources $d$ does not exceed the number of antenna, i.e., $0 \leq$ $d \leq m$.

Notice that, with the relaxation of the orthogonality assumption, eqn. (5) is no longer a rank-1 matrix. As there is no clear relationship between the eigenvectors of $D_{\mathbf{x x}}(t, f)$ and the steering vectors, using singular value decomposition (SVD) on $D_{\mathrm{xx}}(t, f)$, the only information obtained is the number of active sources at that specific point. But which $d$ out of $k$ sources are active is difficult to establish. However, in many applications, it is reasonable to assume that a signal is likely to exist in the system for a certain minimum length of time once detected. In other words, we assume certain temporal continuity of the input sources. This can be justified for example in communications application, where the sources are non-stationary in the sense of their sudden arrival or departure. Thus in a short time interval, a fixed number of sources is present in the system and this number does not exceed the number of the antennae $m$. Based upon this property, the basic idea of our approach is to clustering the neighbouring $t-f$ points that correspond to the same active sources. As a result, the whole t-f plane is partitioned into a certain unknown number of blocks. In each block, at most $m$ sources exist and the adjacent blocks contain different numbers of sources. The important point here is that, within the same block, the active sources remain unchanged. Signal processing can therefore be carried out in a block-by-block manner. Moreover, since there are at most $m$ sources present in each block, the problem of UBSS is transformed into exactly or over determined blind source separation, both of which have neat solutions.

In the implementation, one of the major difficulties is in the way that the t-f plane is partitioned. Let a three source and two antenna system be an example. With an SVD applied to every t-f point $\left(t_{i}, f_{i}\right)$, we obtain set $\Omega_{1}=\left\{\left(t_{i}, f_{i}\right) \mid\right.$ single active source present $\}$ and $\Omega_{2}=$ $\left\{\left(t_{i}, f_{i}\right) \mid\right.$ two active sources present $\}$. It is however difficult to partition $\Omega_{1}$ and $\Omega_{2}$ into appropriate blocks, each of which is contributed by the same active sources. For example, a subset of $\Omega_{2}$ may be contributed by source- 1 and source- 2 while another subset of $\Omega_{2}$ may be contributed by source- 2 and source-3. As it is nearly impossible to know the number of such subsets a priori, when grouping the neighbouring t- $f$ points using classification methods, an advanced clustering technique which does not require the knowledge of the number of clusters is needed. To tackle this problem, a recently proposed clustering technique called self-splitting competitive learning is introduced in our approach, which we briefly explain in the following subsection.

\subsection{Self-Splitting Competitive Learning in UBSS}

The most noticeable advantage of the SSCL algorithm is its capability in automatically determining the number of clusters. The algorithm is proposed from the view of competitive learning in neural networks, in which several prototypes or neurons $\mathbf{p}_{i}$ are competing to 'win' as every member in the data set $Z$ is included in the examination. For our application of UBSS, the data set is the collection of $t-f$ points, i.e., $\Omega_{i}, i=1 \ldots m$. In the ideal situation, each cluster is associated with a prototype at its center. In this sense, the problem of estimating the right number of clusters is effectively the determination of the number of prototypes. The algorithm starts from a single prototype and splits it into more when a certain condition is met. The disadvantage of the conventional competitive learning methods is recognized by the author. That is, one prototype might represent more than one cluster if the number of prototypes is less than the natural number of clusters and, as a result, none of the clus- 
ters could be correctly identified. To avoid this problem, an asymptotic property vector $\mathbf{f}_{i}$ is suggested to guide the learning of each prototype $\mathbf{p}_{i}$. By discriminating the data in $Z$, each prototype is able to represent only one cluster. Specifically, only the data points inside the neighborhood of $\mathbf{p}_{i}$ will contribute to the learning of $\mathbf{p}_{i}$ and the neighborhood is determined with the help of the asymptotic property vector $\mathbf{f}_{i}$. Suppose at some moment during the learning of $\mathbf{p}_{i}$, the member selected from $Z$ is $\widetilde{\mathbf{a}}$. The update equation of $\mathbf{p}_{i}$ is given by

$$
\mathbf{p}_{i}^{\text {new }}=\mathbf{p}_{i}+\alpha_{i}\left(\widetilde{\mathbf{a}}-\mathbf{p}_{i}\right)
$$

where $\alpha_{i}$ is some scalar whose value is related to $\mathbf{p}_{i}, \mathbf{f}_{i}$ and $\widetilde{\mathbf{a}}$. For enough members in $Z$ to be included in the learning of $\mathbf{p}_{i}$, the asymptotic property vector $\mathbf{f}_{i}$ should be initialized far away from $\mathbf{p}_{i}$. During the learning, $\mathbf{f}_{i}$ is also updated such that it approaches $\mathbf{p}_{i}$

$$
\mathbf{f}_{i}^{\text {new }}=\mathbf{f}_{i}+\frac{1}{n_{\mathbf{f}_{i}}} \delta_{i}\left(\widetilde{\mathbf{a}}-\mathbf{f}_{i}\right) \Psi\left(\mathbf{p}_{i}, \mathbf{f}_{i}, \widetilde{\mathbf{a}}\right)
$$

where $\delta_{i}=\left(\frac{\left\|\mathbf{p}_{i}-\mathbf{f}_{i}\right\|_{2}}{\left\|\mathbf{p}_{i}-\widetilde{\mathbf{a}}\right\|_{2}+\left\|\mathbf{p}_{i}-\mathbf{f}_{i}\right\|_{2}}\right)^{2}, \Psi\left(\mathbf{p}_{i}, \mathbf{f}_{i}, \widetilde{\mathbf{a}}\right)$ is a function which gives unity when $\left\|\mathbf{p}_{i}-\mathbf{f}_{i}\right\|_{2} \geq\left\|\mathbf{p}_{i}-\widetilde{\mathbf{a}}\right\|_{2}$ and zero otherwise. The quantity $n_{\mathbf{f}_{i}}$ is the winning counter of $\mathbf{f}_{i}$, whose value is updated by $n_{\mathbf{f}_{i}}^{\text {new }}=n_{\mathbf{f}_{i}}+\delta_{i} \Psi\left(\mathbf{p}_{i}, \mathbf{f}_{i}, \widetilde{\mathbf{a}}\right)$. Upon convergence of $\mathbf{p}_{i}$, the distance between $\mathbf{p}_{i}$ and $\mathbf{f}_{i}$ is smaller than a certain threshold value, which implies no more members within the data set are eligible for the learning of $\mathbf{p}_{i}$.

To determine when the SSCL algorithm should split one of its prototypes, a center property vector $\mathbf{g}_{i}$ is employed for each cluster, which is updated as the exact arithmetic mean of the input data points for which a prototype $\mathbf{p}_{i}$ has so far been the winner. If $\left\|\mathbf{p}_{i}-\mathbf{g}_{i}\right\|_{2}$ is larger than a certain threshold, it suggests that an extra cluster exists which is trying to pull the cluster center from $\mathbf{p}_{i}$ to $\mathbf{g}_{i}$. For non-Gaussian distributed clusters, if $\widetilde{\mathbf{a}}_{j}$ is of dimension $m$, a simple way to determine the threshold is $\frac{1}{50}\left(\max \left(S l_{1}, S l_{2} \ldots S l_{m}\right)\right)$, where $S l_{i}$ is the scale of the $i^{t h}$ coordinate in the $m$ dimension feature space. Once the algorithm decides there should be another new cluster, the current prototype is split into two. One stays at its current location, the other is initialized at some distant location. For more efficiency in the implementation, a distant property vector $\mathbf{r}_{i}$ is used and updated as the learning of $\mathbf{p}_{i}$ continues. But, in contrast to the asymptotic property vector $\mathbf{f}_{i}$, the distant property vector $\mathbf{r}_{i}$ will be updated to a distant location from $\mathbf{p}_{i}$. The algorithm continues until no further cluster is suggested by the splitting criterion. The SSCL algorithm has the advantage of computational simplicity and it is suitable for use in a large data set, which makes it suitable for use in the algorithm.

\subsection{Reconstruction of the Transmitted Sources}

After dividing the t-f plane into blocks, the conventional BSS algorithm (here the JADE algorithm [2]) is applied. The active sources in every time interval are estimated together with the associated steering vectors. But due to the inherent permutational ambiguity of BSS algorithms, the problem of lining up the blocks that correspond to the same source is raised. To tackle this problem, notice from eqn. (1) that each source $s_{i}(t)$ is associated with a steering vector $\mathbf{a}_{i}$ that is kept unchanged for the whole observation period. Hence by classifying the set of estimated steering vectors using for example conventional k-means algorithms, the permutational problem is solved. In summary, a feasible procedure is outlined as followed.

\section{Algorithm for the violation of the t-f orthogonality as- sumption}

Step 1. For computational simplicity, only $\left(t_{i}, f_{i}\right)$ that have sufficient energy are considered. That is, keep $\left(t_{i}, f_{i}\right)$ iff $\left\|D_{\mathbf{x x}}(t, f)\right\|_{F}>\varepsilon$, where $\varepsilon$ is certain threshold and $\|\cdot\|_{F}$ denotes the Frobenius norm. For those selected $\left(t_{i}, f_{i}\right)$, determine the number of active sources $d$ with SVD.

Step 2. For $\Omega_{i}, i=1 \ldots m$, i.e., the regions having the same number of active sources, use SSCL to group neighbouring t-f points into blocks.

Step 3. Apply the JADE algorithm to every block that is associated with more than one active sources, i.e., $\Omega_{i}, i=$ $2 \ldots m$.

Step 4. For the blocks that correspond to the presence of a single source, i.e., $\Omega_{1}$, the steering vectors are estimated as the cluster centers. Apply the FOCUSS algorithm to retrieve the transmitted signal.

Step 5. To line up all the $\mathrm{t}$-f blocks that are corresponding to the same source, all the estimated steering vectors are classified with, for example, the k-means algorithm.

Step 6. Once the permutation becomes known, sum up the estimated signal in different time blocks that are corresponding to the same source.

It should be stressed that besides removing the t-f orthogonality assumption, another advantage of the new approach is that the signals in each t-f block are actually separated in the time-domain. The synthesis stage of signals from their quadratic t-f distribution in [6], which in fact is far from straightforward, is no longer required.

\section{SIMULATION}

We assume a $k=3$ sources and $m=2$ antenna system. The input sources are selected as Gaussian minimum shift keying (GMSK) sources. The two measurement antennae are separated by half of the wavelength. The directions of arrival of the three sources are assumed to be respectively 0 , $\frac{1}{9} \pi$ and $\frac{2}{3} \pi$. Additive white Gaussian noise of $20 \mathrm{~dB}$ signal 


\begin{tabular}{|l|l|}
\hline Source No. & Active time in the system \\
\hline Source-1 & $0-4000 \mathrm{sec}, 5001-7000 \mathrm{sec}, 8001-10000 \mathrm{sec}$ \\
Source-2 & $2001-5500 \mathrm{sec}$ \\
Source-3 & $7101-10000 \mathrm{sec}$ \\
\hline
\end{tabular}

Table 1. Description of three Non-Stationary Source Signals
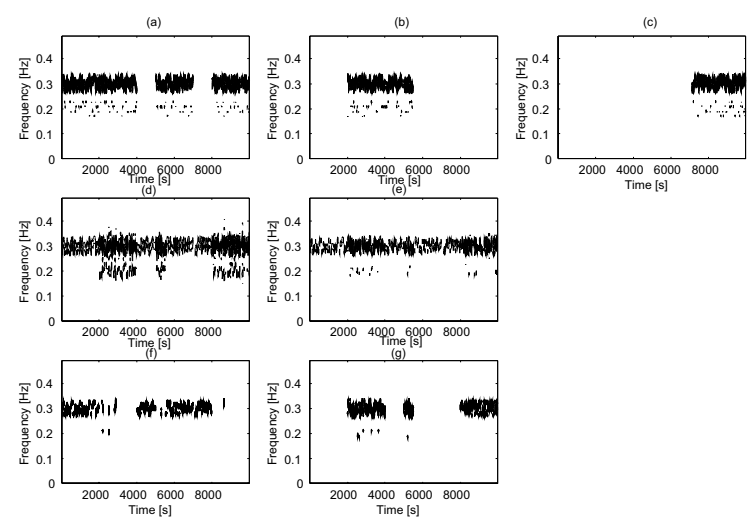

Fig. 1. Source signals, antenna measurement signals and the identified non-overlap and overlap regions (a) Source-1 (b) Source-2 (c) Source-3 (d) Antenna-1 (e) Antenna-2 (f) Region of a single active source (g) Region of two active sources

to noise ratio is present at the antenna measurements. The data rate is $1000 \mathrm{bits} / \mathrm{sec}$ and the sampling rate is $10 \mathrm{ksam}$ ples/sec. The three sources use the same carrier frequency of $3000 \mathrm{~Hz}$. The active times of different source signals are summarized in Table 1. It can be observed that at the time intervals $2001 \mathrm{sec}-4000 \mathrm{sec}, 5001 \mathrm{sec}-5500 \mathrm{sec}$ and $8000 \mathrm{sec}$ $-10000 \mathrm{sec}$, two active sources are simultaneously present in the system, which violates the conventional t-f orthogonality assumption. The $t-f$ representations of the sources are shown in Fig 1(a)-(c) and the antennae measurements are described in 1(d)-(e). With SVD, the proposed algorithm correctly identifies the overlap and non-overlap region, as shown in 1(f)-(g). Using the SSCL method to partition the overlap and non-overlap regions into appropriate blocks, the three GMSK sources are successfully retrieved, as confirmed by their $\mathrm{t}-\mathrm{f}$ representation shown in Fig 2.

\section{CONCLUSION}

We have addressed the problem of underdetermined blind source separation by using $t-f$ analysis. The limitation of the sparsity assumption of the input source in the conventional approach has been highlighted and considerably relaxed. The advanced clustering technique of self-splitting competitive learning was introduced in partitioning the whole
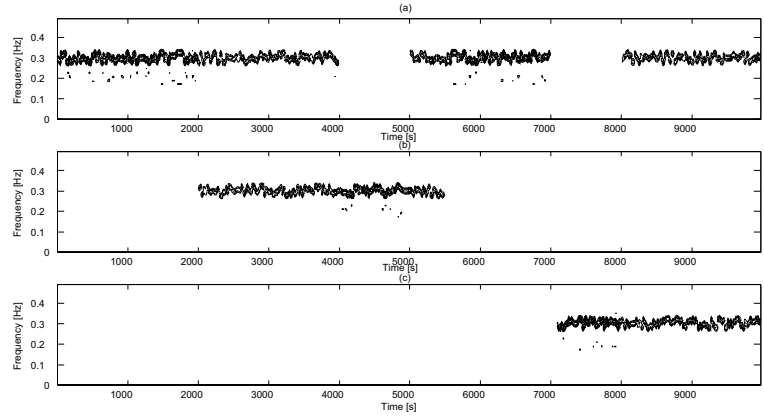

Fig. 2. Successful source separation (a) T-f representation of source-1 (b) T-f representation of source-2 (c) T-f representation of source-3

time frequency domain into appropriate blocks and a new block-based algorithm is therefore proposed and supported by simulation results.

\section{REFERENCES}

[1] P. Bofill and M. Zibulevsky. Underdetermined blind source separation using sparse representation. Signal Processing, 81:2253-2362, 2001.

[2] J.R. Cardoso and A. Souloumiac. Blind beamforming for non-Gaussian signals. IEE Proc. F on Radar and Signal Processing, 140(6):362-370, 1993.

[3] I. F. Gorodnitsky and B. D. Rao. Sparse signal reconstruction from limited data using FOCUSS: A reweighted minimum norm algorithm. IEEE Trans. on Signal Processing, 45(3):600-616, 1997.

[4] A. Jourjine, S. Rickard, and O. Yilmaz. Blind separation of disjoint orthogonal signals: Demixing n sources from 2 mixtures. Proc of IEEE Int. Conf. Acoust. Speech Signal Processing, 5:2985-2988, 2000.

[5] L. Kelva, D. Erdokmus, C. Pantaleon, I. Santamaria, J. Pereda, and J. C. Principe. Underdetermined blind source separation in a time-varying environment. Proc of IEEE Int. Conf. Acoust. Speech Signal Processing, 3:3049-3052, 2002.

[6] L.-T. Nguyen, A. Belouchrain, K. Abed-Meraim, and B. Boashash. Separating more sources than sensors using time-frequency distribution. Sixth International Symposium on Signal Processing and its Applications, 2:583-586, 2001.

[7] Y.-J. Zhang and Z.-Q. Liu. Self-splitting competitive learning: A new on-line clustering paradigm. IEEE Trans. on Neural Networks, 13(2):369-380, 2002. 